\title{
Gradient adsorption of methylene blue and crystal violet onto compound microporous silica from aqueous medium
}

\author{
Yong Li ${ }^{1,2}$, Shifeng Wang ${ }^{1,2,3, *}$, Zichen Shen ${ }^{4}$, Xin Li ${ }^{1}$, Qianyu Zhou ${ }^{1}$, Yaxun Sun ${ }^{1}$, Tingting \\ Wang ${ }^{1}$, Yanfang Liu ${ }^{1}$, Qi Gao ${ }^{1,3, *}$ \\ ${ }^{1}$ Department of Physics, Innovation Center of Materials for Energy and Environment Technologies (i-MEET), \\ College of Science, Tibet University, Lhasa 850000, China; xzuliyong@utibet.edu.cn (Y.L.); \\ 1020772260@qq.com (Z.S.); lixin@utibet.edu.cn（X.L.); zhouqianyu@utibet.edu.cn (Q.Z.); \\ sunyaxun@utibet.edu.cn (Y.S.); wtt@utibet.edu.cn (T.W.); liuyanfang@utibet.edu.cn (F.L.); \\ ${ }^{2}$ Institute of Oxygen Supply, Center of Tibetan Studies (Everest Research Institute), Tibet University, Lhasa \\ 850000, China; \\ ${ }^{3}$ Key Laboratory of Cosmic Rays (Tibet University), Ministry of Education, Lhasa 850000, China \\ ${ }^{4}$ School of Management Engineering, Shandong Jianzhu University, 1000 Fengming Road, Licheng District, Jinan \\ City, Shandong Province 250101, China \\ *Correspondence: wsf@utibet.edu.cn (S.W.), fbc1980@163.com (Q.G.)
}




\section{Supplementary Materials}

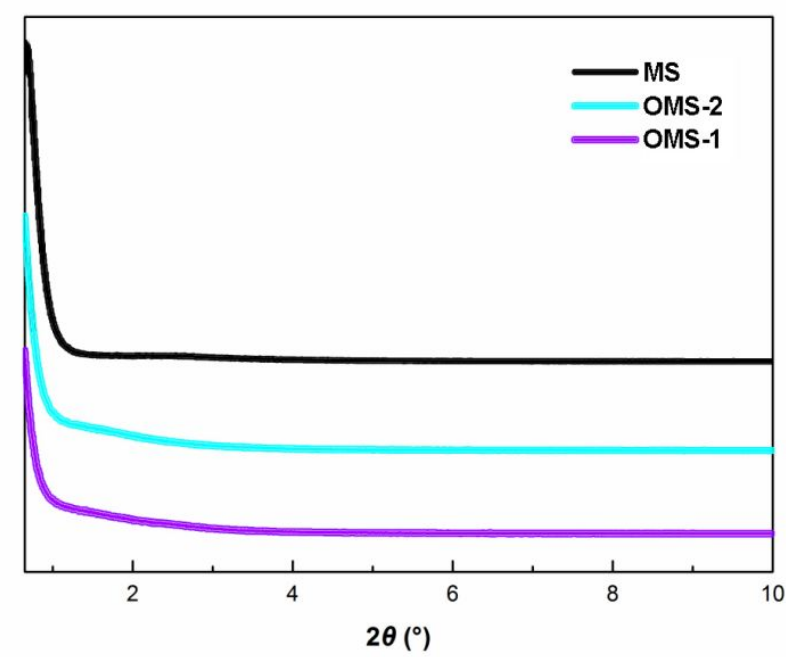

Figure S1. XRD patterns of MS, OMS-1 and OMS-2.
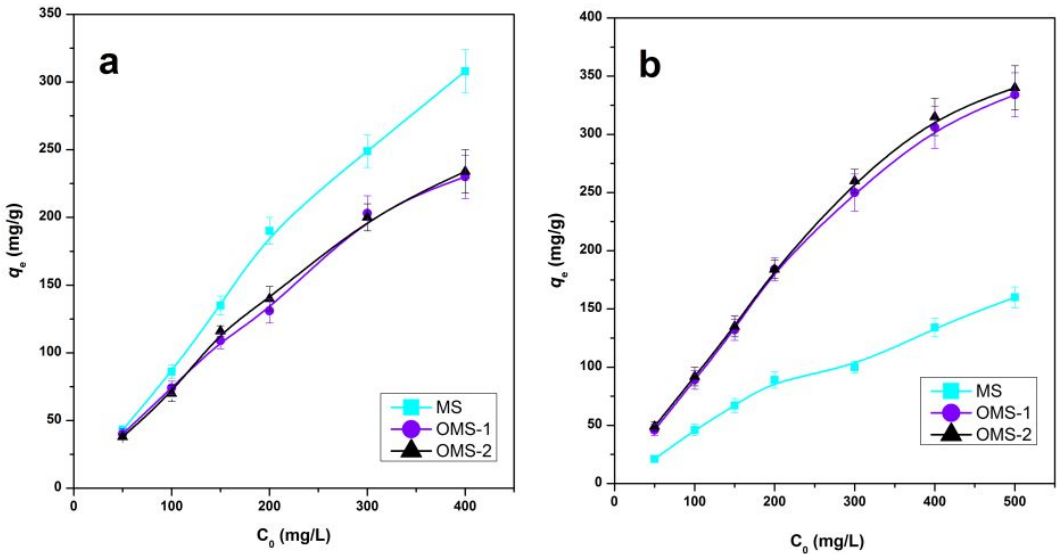

Figure S2. Effect of initial concentration on the removal of MB (a) and CV (b) onto three adsorbents. 

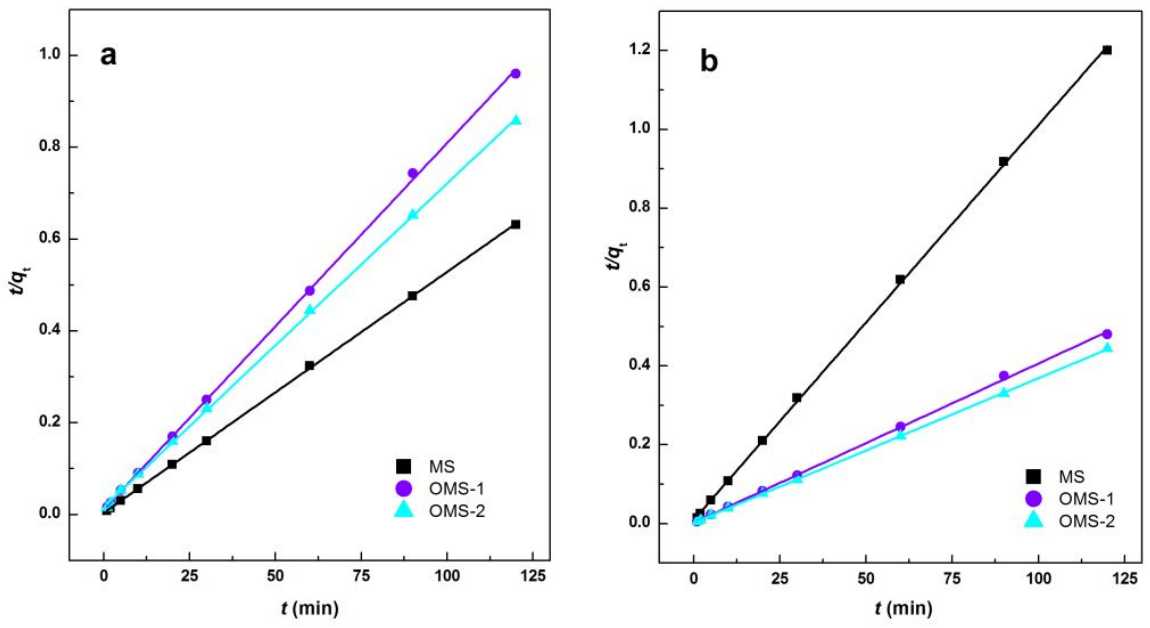

Figure S3. Pseudo-second order plots of MB(a) and CV (b) onto MS, OMS-1 and OMS-2.

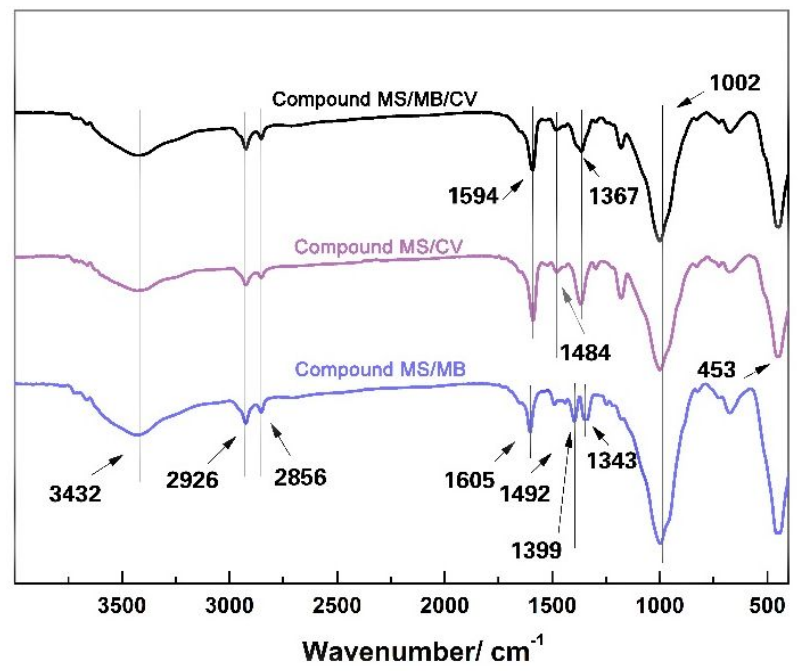

Figure S4. FT-IR spectra of compound MS/MB, compound MS/CV, and compound MS/MB/CV. 

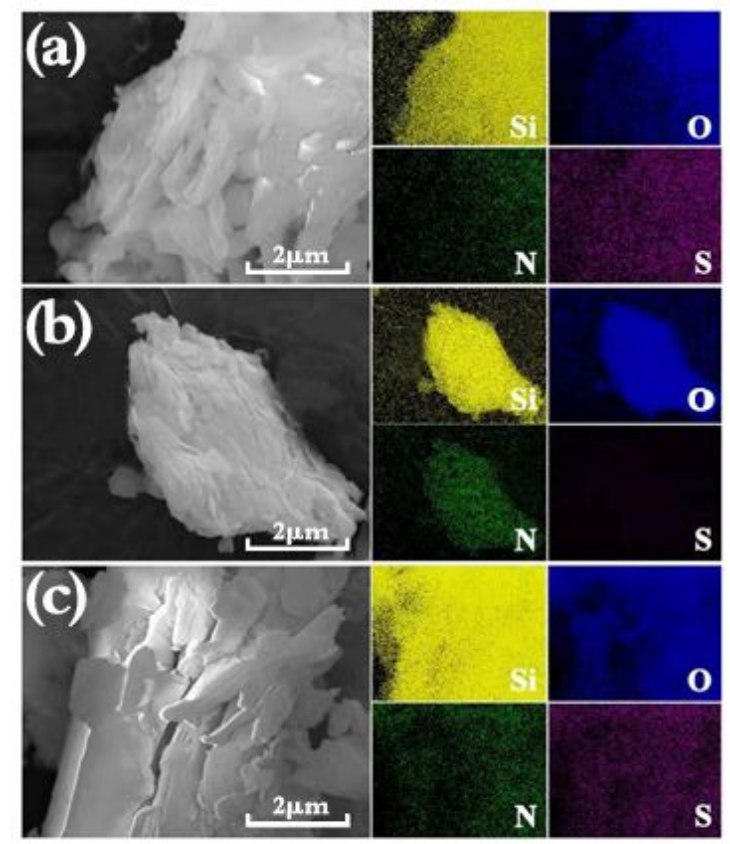

Figure S5. SEM-EDS images of compound MS/MB (a), compound MS/CV (b), and compound $\mathrm{MS} / \mathrm{MB} / \mathrm{CV}(\mathrm{c})$.

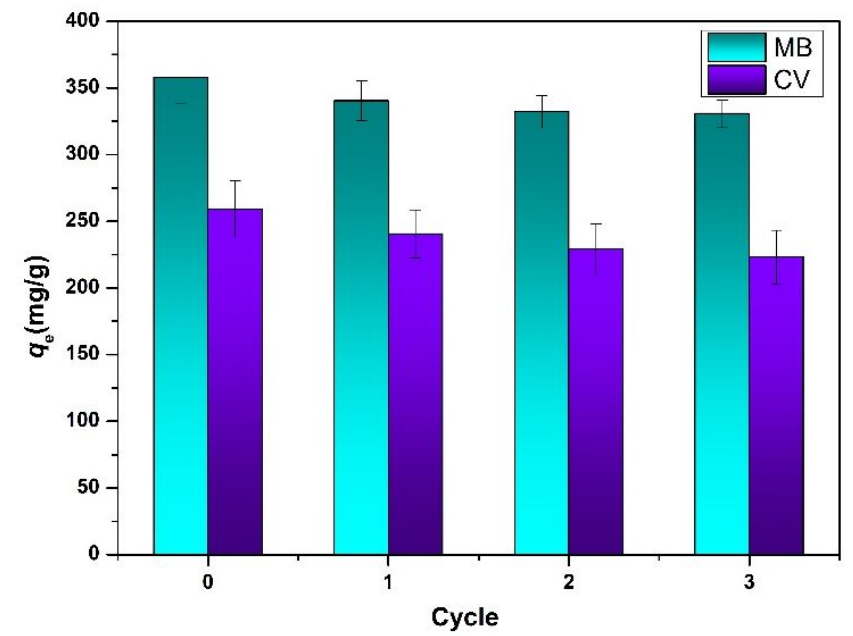

Figure S6. The regenerability of compound MS for adsorption of MB and CV in binary systems. 
Table S1. Elemental analysis data of MS, OMS-1 and OMS-2.

\begin{tabular}{ccccc}
\hline Elemental analysis (\%) & MS & OMS-1 & OMS-2 \\
\hline C & 1.04 & 8.77 & 2.54 \\
H & 1.82 & 2.88 & 0.07 \\
N & 0.02 & 0.18 \\
\hline
\end{tabular}

Table S2. Zeta potentials (mV) of MS, OMS-1 and OMS-2.

\begin{tabular}{cccc}
\hline $\mathbf{p H}$ & MS & OMS-1 & OMS-2 \\
\hline $\mathbf{2}$ & 3.06 & 10.32 & 14.20 \\
$\mathbf{4}$ & -0.25 & 6.88 & 10.06 \\
$\mathbf{6}$ & -0.56 & 3.61 & 6.35 \\
$\mathbf{8}$ & -6.89 & -0.55 & 1.54 \\
$\mathbf{1 0}$ & -8.03 & -5.31 & 0.69 \\
\hline
\end{tabular}


1. The adsorption amount $\left(q_{\mathrm{e}}, \mathrm{mg} \mathrm{g}^{-1}\right)$ was calculated by the following equations:

$$
q_{e}=\frac{C_{0}-C_{e}}{m} V
$$

where $q_{\mathrm{e}}$ is the amount of dyes adsorbed onto adsorbents; $C_{0}\left(\mathrm{mg} \mathrm{g}^{-1}\right)$ and $C_{\mathrm{e}}\left(\mathrm{mg} \mathrm{g}^{-1}\right)$ stands for the initial and equilibrium concentration of dyes, respectively; $m(\mathrm{~g})$ represents the mass of adsorbent and $V(\mathrm{~mL})$ denotes the volume of solution.

2. Adsorption kinetics such as pseudo-first order and pseudo-second order were investigated to set forth the mechanism of dyes on adsorbents. Expressed by the following equations:

pseudo-first order: $\ln \left(q_{e}-q_{t}\right)=\ln q_{e}-k_{1} t$

pseudo-second order: $\frac{t}{q_{t}}=\frac{1}{k_{2} q_{e}^{2}}+\frac{1}{q_{e}} t$

intra-particle diffusion: $q_{\mathrm{t}}=k_{\mathrm{i}} \mathrm{t}^{1 / 2}+C$

where $q_{\mathrm{t}}$ and $q_{\mathrm{e}}$ represents the amounts of phenols adsorbed at time $t$ and equilibrium, respectively; $k_{1}\left(\min ^{-1}\right)$ and $k_{2}\left(\mathrm{~g}(\mathrm{mg} \mathrm{min})^{-1}\right)$ stands for rate constants. $k_{\text {id }}\left(\mathrm{mg} \mathrm{g}^{-1} \min ^{-1 / 2}\right)$ is the rate constant of the intra-particle diffusion kinetic model, the values of $C$ and $k_{\text {id }}$ can be determined from the intercept and slope of the linear plotted of $q_{\mathrm{t}}$ against $\mathrm{t}^{1 / 2}$, respectively.

3. Langmuir and Freundlich isotherm were vetted for further study of the adsorption mechanism of two dyes onto three adsorbents. The adsorption process of Langmuir isotherm fitting. Equations for the two isotherms were shown below:

$$
\text { Langmuir isotherm: } \begin{aligned}
\frac{C_{e}}{q_{e}} & =\frac{1}{K_{L} q_{m}}+\frac{C_{e}}{q_{m}} \\
R_{L} & =\frac{1}{1+K_{L} C_{0}}
\end{aligned}
$$

Freundlich isotherm: $\ln q_{e}=\ln K_{F}+\frac{1}{n} \ln C_{e}$ 
Redlich-Peterson: $q_{e}=\frac{A C_{e}}{1+b C_{e}^{g}}$

where $C_{\mathrm{e}}\left(\mathrm{mg} \mathrm{L}^{-1}\right)$ was the concentration of adsorbate at equilibrium, $q_{\mathrm{e}}\left(\mathrm{mg} \mathrm{L}^{-1}\right)$ symbolized the equilibrium adsorption capacity, $q_{\mathrm{m}}\left(\mathrm{mg} \mathrm{L}^{-1}\right)$ meant the largest monolayer adsorption capacity of phenols in theory, $K_{\mathrm{L}}\left(\mathrm{L} \mathrm{mg}^{-1}\right)$ represented the Langmuir equilibrium constant which related to affinity of binding sites and energy of adsorption, $R_{\mathrm{L}}$ was defined as the separation factor. $K_{\mathrm{F}}(\mathrm{mg}$ $\left.\mathrm{g}^{-1}\right)$ and $n$ belonged to Freundlich constant; $\mathrm{A}\left(\mathrm{L} \mathrm{g} \mathrm{g}^{-1}\right), \mathrm{b}\left(\left(\mathrm{L} \mathrm{mg}^{-1}\right)^{\mathrm{g}}\right)$ and $\mathrm{g}$ (Eq. 7) were allocated as the constants of Redlich-Peterson model, respectively.

4. The energy variations of the adsorption process were measured by thermodynamic parameters $\left(\Delta G^{\circ}, \Delta H^{\circ}\right.$ and $\left.\Delta S^{\circ}\right)$, being calculated from the adsorption experiments at various temperatures using the equations below:

$$
\begin{aligned}
& \ln K_{L}=\frac{\Delta S^{\natural}}{R}-\frac{\Delta H^{\natural}}{R T} \\
& \Delta G^{\circ}=\Delta H^{\circ}-T \Delta S^{\circ}
\end{aligned}
$$

where $K_{\mathrm{L}}\left(\mathrm{L} \mathrm{mol}^{-1}\right)$ fell with the Langmuir constant, $R\left(8.314 \mathrm{~J} \mathrm{~mol}^{-1} \mathrm{~K}^{-1}\right)$ was the gas constant, $T$ $(\mathrm{K})$ was on behalf of the reaction temperature in Kelvin. 